\title{
ON THE MINIMA OF YANG-MILLS FUNCTIONALS
}

\author{
CHAOHAO GU
}

Dedicated to the author's teacher Professor Buchin Su

In a previous paper [1] we found some lower bounds of the Yang-Mills functional on the tangential bundle over a 4-dimensional oriented manifold among all possible metrics with the Christoffel connections as the gauge potentials [2]. In this paper the results are generalized to vector bundles over a 4-dimensional oriented manifolds, provided the structure group (gauge group) $G$ is compact and its Lie algebra $g$ is nonsimple. Some lower bounds of the Yang-Mills functional are obtained and several cases for which the lower bounds are actually the absolute minimums are listed. In particular it is seen that for the Einstein manifolds or the conformally flat manifolds with zero scalar curvature the Yang-Mills functional attains its absolute minimum among all possible metrics on the manifold and all possible connections on the tangential bundles.

Let $G$ be a compact Lie group, and suppose that its Lie algebra $g$ be the direct sum of two Lie algebras

$$
g=g_{1}+g_{2}
$$

An arbitrary element $\alpha$ of $g$ may be written in the form $\alpha=\left(\alpha_{1}, \alpha_{2}\right)$ with $\alpha_{1} \in g_{1}, \alpha_{2} \in g_{2}$. We define a linear mapping * on the Lie algebra $g$ to itself by

$$
*\left(\alpha_{1}, \alpha_{2}\right)=\left(\alpha_{1},-\alpha_{2}\right) \text {. }
$$

Then the following relations evidently hold:

$$
\begin{gathered}
*^{2}=I, \\
\text { (b) }[\alpha, * \beta]=\left(\left[\alpha_{1}, \beta_{1}\right],\left[\alpha_{2},-\beta_{2}\right]\right)=*([\alpha, \beta]),
\end{gathered}
$$

(c) $\langle * \alpha, * \beta\rangle=\left\langle\alpha_{1}, \beta_{1}\right\rangle+\left\langle-\alpha_{2},-\beta_{2}\right\rangle=\langle\alpha, \beta\rangle$,

Received June 6, 1979. The author is grateful to Professor C. N. Yang for valuable discussions, and also to the State University of New York at Stony Brook for its hospitality. 
where $[$,$] and \langle$,$\rangle are the commutator and the invariant inner product$ respectively.

Theorem 1. If $g$ is a Lie algebra, and there exists a linear mapping $*: g \rightarrow g$ such that (a) and (b) hold, then $g$ is the direct sum of the subalgebras, except for the trivial case $*= \pm I$.

Proof. Let

$$
g_{1}=\{\alpha+* \alpha \mid \alpha \in g\}, \quad g_{2}=\{\alpha-* \alpha \mid \alpha \in g\} .
$$

Since * is nontrivial, $g_{1}$ and $g_{2}$ are both nontrivial subspaces.

From (b) it is easily seen that

$$
\begin{aligned}
& {[\alpha+* \alpha, \beta+* \beta]=([\alpha, \beta]+[* \alpha, \beta])+*([\alpha, \beta]+[* \alpha, \beta]),} \\
& {[\alpha-* \alpha, \beta-* \beta]=([\alpha, \beta]-[* \alpha, \beta])-*([\alpha, \beta]-[* \alpha, \beta]) .}
\end{aligned}
$$

Hence $g_{1}$ and $g_{2}$ are both subalgebras. Moreover, from (a) and (b) we have

$$
[\alpha+* \alpha, \beta-* \beta]=[\alpha, \beta]+[* \alpha, \beta]-[\alpha, * \beta]-[* \alpha, * \beta]=0 \text {, }
$$

i.e., the elements of $g_{1}$ and those of $g_{2}$ are commutative. Finally, if $\gamma \in$ $g_{1} \cap g_{2}$, then $* \gamma= \pm \gamma$ which implies that $\gamma=0$. Hence $g_{1} \cap g_{2}=\{0\}$, and the theorem is proved.

The mapping $*$ is called the generalized dual operator, since it contains the usual duality in $R^{4}$ as a particular case: Let $g$, be the Lie algebra so (4) formed by $4 \times 4$ skew-symmetric matrices $L=\left(l_{a b}\right), a, b=1, \cdots, 4$. Then $g_{1}$ and $g_{2}$ are sets of self-dual and antiself-dual matrices respectively, i.e.,

$$
\begin{array}{lll}
L \in g_{1} & \text { iff } & l_{a b}=\frac{1}{2} \varepsilon_{a b c d} l_{c d} \\
L \in g_{2} & \text { iff } & l_{a b}=-\frac{1}{2} \varepsilon_{a b c d} l_{c d} .
\end{array}
$$

The * is the dual operator

$$
* L=\left(l_{a b}^{*}\right)
$$

with

$$
l_{a b}^{*}=\frac{1}{2} \varepsilon_{a b c d} l_{c d} .
$$

Now let $M$ be a 4-dimensional oriented Riemannian manifold, and $E \rightarrow M$ a vector bundle over $M$ with structure group $G$. Let $b$ be a connection or gauge potential on $E$. In a small patch $b$ is expressed as $g$-valued 1-form

$$
b=b_{\lambda}(x) d x^{\lambda}
$$

and the field strength is the $g$-valued 2-form:

$$
\begin{aligned}
F & =d b+\frac{1}{2}[b, b]=\frac{1}{2} F_{\lambda \mu} d x^{\lambda} \wedge d x^{\mu} \\
& =\frac{1}{2}\left(\partial_{\lambda} b_{\mu}-\partial_{\mu} b_{\lambda}+\left[b_{\lambda}, b_{\mu}\right]\right) d x^{\lambda} \wedge d x^{\mu} .
\end{aligned}
$$


Define

$$
\begin{aligned}
I_{W} & =\int_{M}\left\langle F_{p q}, F^{p q}\right\rangle d V=\int_{M} K_{W} d V, \\
I_{E} & =\frac{1}{2} \int_{M}\left\langle F_{p q}, * F_{r s}\right\rangle \varepsilon^{p q r s} d V=\int_{M} K_{E} d V, \\
I_{P} & =\frac{1}{2} \int_{M}\left\langle F_{p q}, F_{r s}\right\rangle \varepsilon^{p q r s} d V=\int_{M} K_{P} d V, \\
I_{Q} & =\int_{M}\left\langle F_{p q}, * F^{p q}\right\rangle d V=\int_{M} K_{Q} d V
\end{aligned}
$$

where $I_{W}$ is the Yang-Mills functional, and * the generalized dual operator. $I_{P}$ is the integral of the 2 nd Chern class or 1st Pontrjagen class up to a constant factor. $I_{E}$ is the generalization of the Euler's characteristic number and is also a topological invariant, if the manifold is compact and free of boundary. This is a consequence of the Chern-Weil's theorem [3], since the bilinear form $f(\alpha, \beta)=\langle\alpha, * \beta\rangle$ on $g \times g$ is symmetric and invariant. In general $I_{Q}$ is not a topological invariant. However, for the tangential bundle $I_{P}=I_{Q}$ if the connection is the Christoffel connection of the Riemannian metric.

Define the inner product of two $g$-valued 2 -form at each point of $M$

$$
F \cdot \Phi=\left\langle F_{p q}, \Phi^{p q}\right\rangle \text {. }
$$

It is easy to verify that

$$
\begin{aligned}
F \cdot F & =F^{* *} \cdot F^{* *}=F^{* \cdot} \cdot F^{* *}=F^{* *} \cdot F^{* *}=K_{W}, \\
F \cdot F^{* *} & =F^{*} \cdot F^{* *}=K_{P}, \\
F \cdot F^{*} \cdot & =F^{* *} \cdot F^{* *}=K_{Q} \\
F \cdot F^{* *} & =F^{*} \cdot F^{* *}=K_{E} .
\end{aligned}
$$

Here the 1 st $*$ is the generalized dual operator, and the 2 nd $*$ is the operator (6).

Let

$$
\begin{aligned}
F^{++} & =\frac{1}{4}\left(F^{* *}+F^{* \cdot}+F^{* *}+F^{* *}\right), \\
F^{-+} & =\frac{1}{4}\left(F^{*}-F^{* \cdot}+F^{* *}-F^{* *}\right), \\
F^{+-} & =\frac{1}{4}\left(F^{*}+F^{* \cdot}-F^{* *}-F^{* *}\right), \\
F^{--} & =\frac{1}{4}\left(F^{*}-F^{* \cdot}-F^{* *}+F^{* *}\right) .
\end{aligned}
$$


We have

$$
\begin{aligned}
F^{++} \cdot F^{++} & =\frac{1}{4}\left(K_{W}+K_{Q}+K_{P}+K_{E}\right), \\
F^{+-} \cdot F^{+-} & =\frac{1}{4}\left(K_{W}+K_{Q}-K_{P}-K_{E}\right), \\
F^{++} \cdot F^{++} & =\frac{1}{4}\left(K_{W}-K_{Q}+K_{P}-K_{E}\right), \\
F^{--} \cdot F^{--} & =\frac{1}{4}\left(K_{W}-K_{Q}-K_{P}+K_{E}\right), \\
F^{++} \cdot F^{+-} & =F^{++} \cdot F^{-+}=F^{++} \cdot F^{--}=F^{+-} \cdot F^{++} \\
& =F^{+-} \cdot F^{--}=F F^{-+} \cdot F^{--}=0 .
\end{aligned}
$$

Using these identities we can easily obtain

Theorem 2. If $A, B, C, D$ are arbitrary constants and not all zero, then

$$
\begin{aligned}
I_{W}=\frac{1}{A^{2}+B^{2}+C^{2}+D^{2}}\{ & \int_{M}\left(A F^{++}+B F^{+-}+C F^{-+}+D F^{--}\right)^{2} d V \\
& +\left[\left(-I_{P}-I_{Q}-I_{E}\right) A^{2}+\left(I_{P}+I_{E}-I_{Q}\right) B^{2}\right. \\
+ & \left.\left.\left(I_{E}-I_{P}+I_{Q}\right) C^{2}+\left(I_{Q}+I_{P}-I_{E}\right) D^{2}\right]\right\} .
\end{aligned}
$$

Further, we have

Theorem 3.

$$
\text { (a) } \begin{array}{r}
I_{W} \geqslant \max \left\{\left(-I_{P}-I_{Q}-I_{E}\right),\left(I_{P}+I_{E}-I_{Q}\right),\left(I_{E}-I_{P}+I_{Q}\right)\right. \\
\left.\left(I_{P}+I_{Q}-I_{E}\right)\right\} .
\end{array}
$$

(b) The equality sign of the above inequality holds if and only if at least one of $\mathrm{F}^{++}, \mathrm{F}^{+-}, \mathrm{F}^{-+}, \mathrm{F}^{++}$is zero.

Proof. (a) follows directly from the identity (17).

(b) Suppose the equality sign holds, say $I_{W}=-I_{P}-I_{Q}-I_{E}$. Let $A=1$. Then $B=C=D=0$, and we have $F^{++}=0$. Conversely, if $F^{++}=0$, the same set of $A, B, C, D$ gives $I_{W}=-I_{P}-I_{Q}-I_{E}$. Moreover, from (18) it follows that

$$
\begin{aligned}
& -I_{P}-I_{Q}-I_{E} \geqslant I_{P}+I_{E}-I_{Q} \\
& -I_{P}-I_{Q}-I_{E} \geqslant I_{E}-I_{P}+I_{Q} \\
& -I_{P}-I_{Q}-I_{E} \geqslant I_{P}+I_{Q}-I_{E}
\end{aligned}
$$

and hence (b) is proved.

Now we can list several cases in which the Yang-Mills functional attains its possible minimal value for given values of $I_{P}, I_{E}$ and $I_{Q}$ : 


\begin{tabular}{|c|c|c|c|}
\hline No & $F$ & $I_{P}, I_{Q}, I_{E}$ & $I_{W}$ \\
\hline 1 & $F^{++}=0$ & $-I_{Q}-I_{E}>0,-I_{P}-I_{E}>0,-I_{P}-I_{Q}>0$ & $-I_{P}-I_{Q}-I_{E}$ \\
2 & $F^{+-}=0$ & $I_{P}+I_{E}>0,-I_{Q}+I_{P}>0,-I_{Q}+I_{E}>0$ & $I_{P}+I_{E}-I_{Q}$ \\
3 & $F^{-+}=0$ & $I_{E}+I_{Q}>0, I_{Q}-I_{P}>0, I_{E}-I_{P}>0$ & $I_{E}+I_{Q}-I_{P}$ \\
4 & $F^{--}=0$ & $I_{P}+I_{Q}>0, I_{P}-I_{E}>0, I_{Q}-I_{E}>0$ & $I_{P}+I_{Q}-I_{E}$ \\
1,2 & $F^{++}=F^{+-}=0$ & $-I_{Q}>I_{E}=-I_{P}>I_{Q}$ & $-I_{Q}$ \\
1,3 & $F^{++}=F^{-+}=0$ & $-I_{P}>I_{E}=-I_{Q}>I_{P}$ & $-I_{P}$ \\
1,4 & $F^{++}=F^{--}=0$ & $-I_{E}>I_{P}=-I_{Q}>I_{E}$ & $-I_{E}$ \\
2,3 & $F^{+-}=F^{-+}=0$ & $I_{E}>I_{P}=I_{Q}>-I_{E}$ & $I_{E}$ \\
2,4 & $F^{+-}=F^{--}=0$ & $I_{P}>I_{E}=I_{Q}>-I_{P}$ & $I_{P}$ \\
3,4 & $F^{++}=F^{--}=0$ & $I_{Q}>I_{P}=I_{E}>-I_{Q}$ & $I_{Q}$ \\
$2,3,4$ & $F^{+-}=F^{-+}=F^{--}=0$ & $I_{E}=I_{P}=I_{Q}>0$ & $I_{P}$ \\
$1,3,4$ & $F^{++}=F^{-+}=F^{--}=0$ & $-I_{P}=I_{Q}=-I_{E}>0$ & $-I_{P}$ \\
$1,2,4$ & $F^{++}=F^{+-}=F^{--}=0$ & $-I_{Q}=-I_{E}=I_{P}>0$ & $I_{P}$ \\
$1,2,3$ & $F^{++}=F^{+-}=F^{-+}=0$ & $-I_{Q}=-I_{P}=I_{E}>0$ & $-I_{P}$ \\
\hline
\end{tabular}

In a general manifold the values of $I_{E}, I_{P}, I_{Q}$ place some constraints on both the Riemannian metric and the connection. For compact manifolds without boundary, $I_{E}$ and $I_{P}$ are topological invariants. So the cases $(1,3)$, $(1,4),(2,3)(2,4)$ (and hence $(2,3,4)(1,3,4)(1,2,4),(1,2,3))$ are more interesting than the other cases. In particular, consider the tangential bundle $E$ over an oriented compact manifold $M$ without boundary. If $M$ admits an Einstein metric or a conformally flat metric with zero scalar curvature, then we have cases $(2,3)$ or $(1,4)$ respectively. Hence the Yang-Mills function attains absolute minimum in both cases.

Corollary. The Einstein metric or the conformally flat metric with zero scalar curvature on the compact manifold without boundary minimizes the Yang-Mills functional on the tangential bundle, provided the connection is the Christoffel connection of the metric.

Remark. If the Lie algebra $g$ is decomposable to a direct sum of more than two components, then more detail results can be obtained in the same way.

\section{References}

[1] C. H. Gu, H. S. Hu, D. Q. Li, C. L. Shen, Y. L. Xin \& C. N. Yang, Riemannian spaces with local duality and gravitational instantons, Scientia Sinica 21 (1978) 475.

[2] C. N. Yang, Integral formalism for gauge fields, Lett. Phys. Rev. 33 (1974) 445-447.

[3] S. S. Chern, Geometry of characteristic classes, Proc. 13th Biennial Sem. Canadian Math. Congress, Vol. 1, 1972, 1-40.

State University OF NeW York, Stony BrooK FUdAN UNIVERSITY, SHANGHAI 
